\title{
Noteworthy records of corticioid fungi from Ichnia National Nature Park
}

\author{
Mariia V. SHEVCHENKO \\ M.G. Kholodny Institute of Botany, National Academy of Sciences of Ukraine \\ 2, Tereshchenkivska Str., Kyiv 01004, Ukraine \\ Shevchenko_Mariya@ex.ua
}

Shevchenko M.V. Noteworthy records of corticioid fungi from Ichnia National Nature Park. Ukr. Bot. J., 2018, 75(1): 77-83.

Abstract. Corticioid fungi constitute a heterogeneous group in terms of origin; they belong to various orders of Agaricomycetes (Basidiomycota). According to the available data, ca 280 species of this group have been so far recorded in Ukraine. As compared to other countries of Europe, diversity of corticioid fungi in Ukraine has been insufficiently studied. We made observations and collected corticioid fungi in June-October, 2016 and in March-October, 2017 in forests and woods of Ichnia National Nature Park (Ichnia District, Chernihiv Region, Ukraine). Upon identification of the herbarium specimens collected over the study area, we recorded 90 species of corticioid fungi. This article reports three noteworthy records of corticioid fungi. Two species, Amylocorticium canadense and Tubulicrinis glebulosus, are new records for Ukraine; another one, Aleurodiscus dextrinoideocerussatus, is a rare species both in Ukraine and globally, collected in our country for the second time, and a new record for the Forest-Steppe zone. The specimens were examined in 5\% aqueous potassium hydroxide solution, Melzer's reagent and Lactophenol cotton blue. For all the species, we provide descriptions of the examined specimens, along with the original photos, substrate specialization and global distribution details. The collected specimens are deposited in the Mycological Herbarium of the M.G. Kholodny Institute of Botany, National Academy of Sciences of Ukraine $(K W-M)$.

Keywords: Ukraine, Left-Bank Forest-Steppe, Amylocorticiales, Hymenochaetales, Russulales, Aleurodiscus dextrinoideocerussatus, Amylocorticium canadense, Tubulicrinis glebulosus

\section{Introduction}

Corticioid fungi (Basidiomycota) constitute a group of fungi, which is heterogeneous in terms of origin. Fungi of this group form morphologically similar basidiocarps due to convergence. Basidiomata of these fungi are fully resupinate or effuse-reflexed, hymenophore shape varies from smooth to almost poroid, reticulate or irpicoid and long-toothed (Jülich, Stalpers, 1980; Yurchenko, 2006; Zmitrovich, 2008).

Most of corticioid fungi are xylosaprotrophs. Thus, along with polypores they are important components of forest ecosystems, being the major fungal destructors, degrading lignin and cellulose in the nature. Insignificant number of these fungi are pathogens of vascular plants and mycorrhiza-forming fungi (Yurchenko, 2006).

Nowadays, about 1,800 species of corticioid fungi are known in the world (Mueller et al., 2006). In the temperate zone of Europe, 611 species of them are reported (Mueller et al., 2006). Currently, ca. 280 fungi of this group have been recorded in Ukraine (Akulov

(C) M.V. SHEVCHENKO, 2018 et al., 2003; Küffer et al., 2004; Usichenko, 2009; Bernicchia, Gorjón, 2010; Ordynets, Yurchenko, 2010; Ordynets, Akulov, 2011; Ordynets et al., 2011). As compared to other countries of Europe, the corticioid fungi diversity in Ukraine is still insufficiently studied (Akulov et al., 2003; Ordynets, Yurchenko, 2006). Corticioid fungi of the Left-Bank Forest-Steppe have been studied very unevenly. For instance, there are about 100 species of these fungi known for the Kharkiv ForestSteppe, the best-studied area in this zone (Akulov et al., 2003; Usichenko, 2009). Before we started our surveys, no records of corticioid fungi distribution were reported from Ichnia National Nature Park. Therefore, further examination of these fungi in Ukraine is desirable.

\section{Materials and methods}

Materials used in this research comprise corticioid fungi collected in June-October, 2016 and in MayOctober, 2017 in the forests of Ichnia National Nature Park. Ichnia National Nature Park is located in the north of Ukraine, in Ichnia District, Chernihiv Region. The park's total area is $9,665.8$ hectares. This 
area, according to the zoning proposed by V. Heluta (1989) in Flora Gribov Ukrainy, belongs to the LeftBank Forest-Steppe zone. Unique characters of the natural vegetation in Ichnia National Nature Park are determined by its position in the northern part of the Left-Bank Forest-Steppe. In terms of the vegetation cover, the forests prevail, as forest coverage constitutes $83 \%$. The forestlands are of rather limited size, being located relatively evenly all over the park's territory. Among the peculiar features of Ichnia National Nature Park, overlapping of oak, hornbeam and lime forest areas is worth mentioning (Zhyhalenko, 2009). The park's hornbeam forests make up the eastern boundary of the natural range of this species (Zhyhalenko, 2009). Pine forests prevail here, although large areas are also covered with birch and oak forests. Pine forests are located mainly in the northern part of the park, while oak and hornbeam-oak forests occupy the central and southeastern parts, and lime forests are found in the western part of the park. The alder forests occur along the beds of the Udai and Ichenka rivers and their tributaries. Significant areas are covered with pine forest stands (Zhyhalenko, 2009).

The specimens were examined in 5\% aqueous potassium hydroxide solution, Melzer's reagent and Lactophenol cotton blue. For identification of the specimens, a number of monographs and articles on systematics and diversity of corticioid fungi were used (Eriksson, Ryvarden, 1973; Eriksson, Weresub, 1974; Jülich, Stalpers, 1980; Núñez, Ryvarden, 1997; Ghobad-Nejhad et al., 2009; Bernicchia, Gorjón, 2010). The species distribution was analyzed based on the checklist of aphyllophoroid fungi of Ukraine (Akulov et al., 2003) and various publications on corticioid fungi of Ukraine (Küffer et al., 2004; Usichenko, 2009; Ordynets, Yurchenko, 2010; Ordynets et al., 2011).

The nomenclature of the species follows the Index Fungorum database [http://www.indexfungorum. org/names/names.asp]. The collected specimens are deposited in Mycological Herbarium of the M.G. Kholodny Institute of Botany, National Academy of Sciences of Ukraine $(K W-M)$.

\section{Results and discussion}

Among the herbarium specimens we collected over the studied area, 90 species of corticioid fungi were identified. Some of them had been reported earlier (Shevchenko, 2017). This article reports details about two species of corticioid fungi, Amylocorticium canadense and Tubulicrinis glebulosus, which are new for Ukraine, and one species, Aleurodiscus dextrinoideocerussatus, which is rare in Ukraine. These species belong to various orders of Agaricomycetes.

The reported taxa are listed in systematic order. The nomenclature is followed by information on morphology, specimens examined, ecological peculiarities and distribution of the species. The original photographs of specific macro- and micromorphological structures are provided for each species.

Amylocorticiales K.H. Larss., Manfr. Binder \& Hibbett

\section{Amylocorticiaceae Jülich}

Amylocorticium canadense (Burt) J. Erikss. \& Weresub, Fungi Canadenses, no. 127 (Ottawa): no. 45. 1974 (Fig. 1; $a, b$ )

Basidioma resupinate, effused, adherent, soft to submembranaceous, up to $0.5 \mathrm{~mm}$ thick. Hymenial surface smooth, almost easily detachable in small pieces from the subiculum, cream to very pale yellow, slightly cracked on drying, immediately darkening when treated with $\mathrm{KOH}$. Subiculum sulphureous yellowish, yellowish-brown, tomentose or fimbriate, almost black when treated with $\mathrm{KOH}$. Margin determinate, abrupt or thinning out, smooth to finely byssoid, yellow to yellowish-brown, lighter to concolorous with the subiculum. Hyphal system monomitic, all hyphae with fibulate septa; subhymenial ones $2.5-3.5(4.0) \mu \mathrm{m}$ in diameter, vertically oriented, compactly arranged, thinwalled, hyaline; subicular ones loosely intertwined, regular, 3.5-4.5 $\mu \mathrm{m}$, with thickening wall, hyaline to yellowish. Cystidia absent. Basidia narrowly clavate to subcylindrical, (15)20-25 × 4-5 $\mu \mathrm{m}$, hyaline; 4 sterigmata up to $4 \mu \mathrm{m}$ long. Basidiospores narrowly ellipsoid to cylindrical, slightly allantoid, usually biguttulate with two drops towards each end of the spores, in side view often slightly bent, (4.0)4.5$5.0(6.2) \times 1.6-2.5 \mu \mathrm{m}$, thin-walled, weakly amyloid, not cyanophilous.

Specimens examined: Ukraine, Chernihiv Region, Ichnia District, Ichnia National Nature Park, Peliukhivka village, forest stand of Pinus sylvestris L., August 07, 2016, $K W-M$ 70877, 70878; outskirts of Peliukhivka village, mixed forest, on fallen branches of Pinus sylvestris, August 07, 2016, KW-M 70876.

Ecological peculiarities: on fallen branches of angiosperms (Bernicchia, Gorjón, 2010) and gymnosperms (Abies Mill., Picea A. Dietr., Pinus L., Tsuga (Endl.) Carrière) (Gilbertson, Lindsey, 1989; Boidin, Gilles, 1990). 

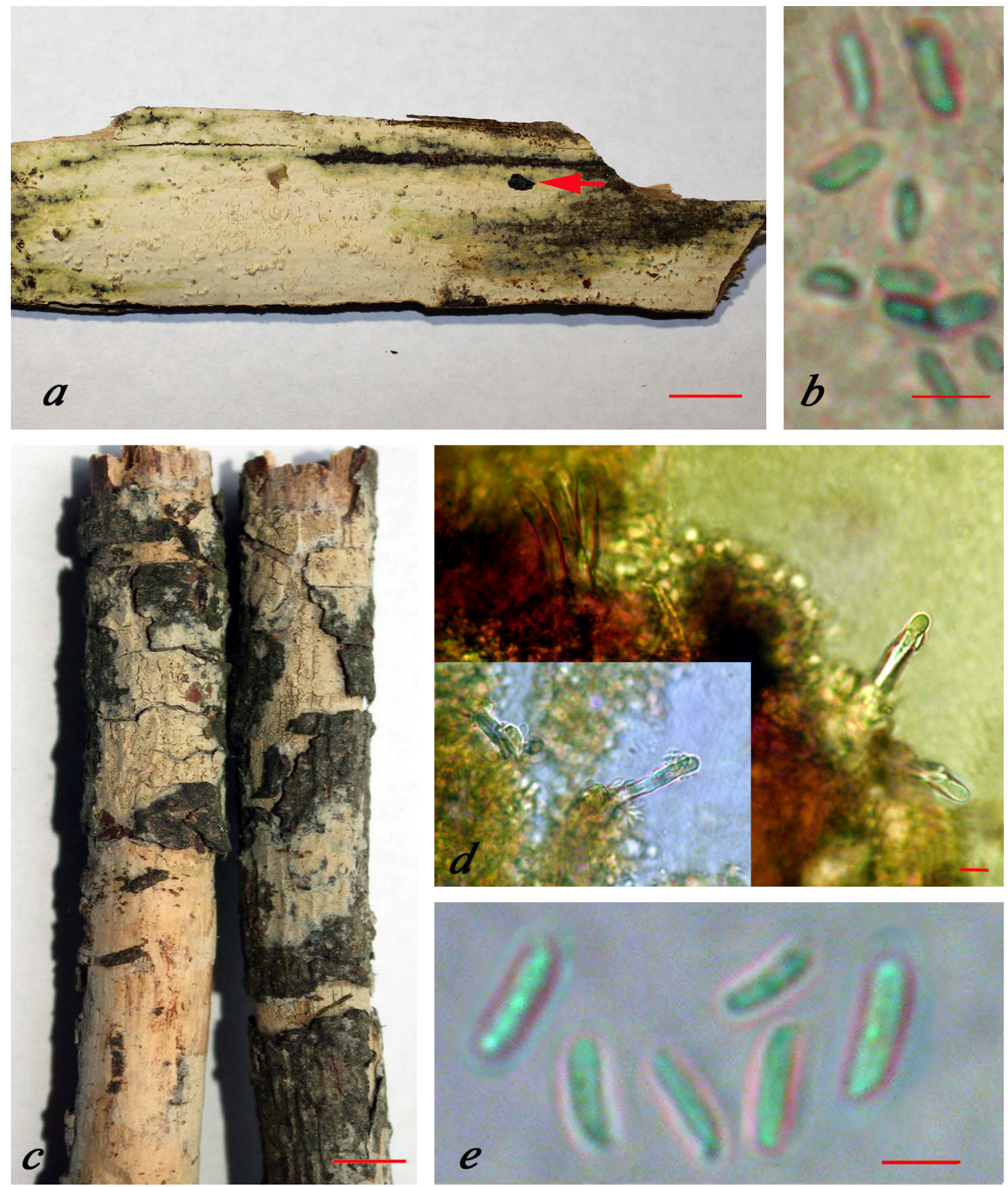

Fig. 1. Macroscopic and microscopic peculiarities of Amylocorticium canadense $K W-M 70877(a, b)$ and Tubulicrinis glebulosus $K W-M 70875(c-e) ; a-$ general view of basidioma of $A$. canadense, arrow indicates the location of basidioma darkening when treated with $\mathrm{KOH} ; b, e$ - basidiospores; $c$ - general view of basidioma of T. glebulosus; $d$ - smooth and encrusted lyocystidia (bars: $a, c-1 \mathrm{~cm}, d-20 \mu \mathrm{m} b, e-5 \mu \mathrm{m}$ ) 

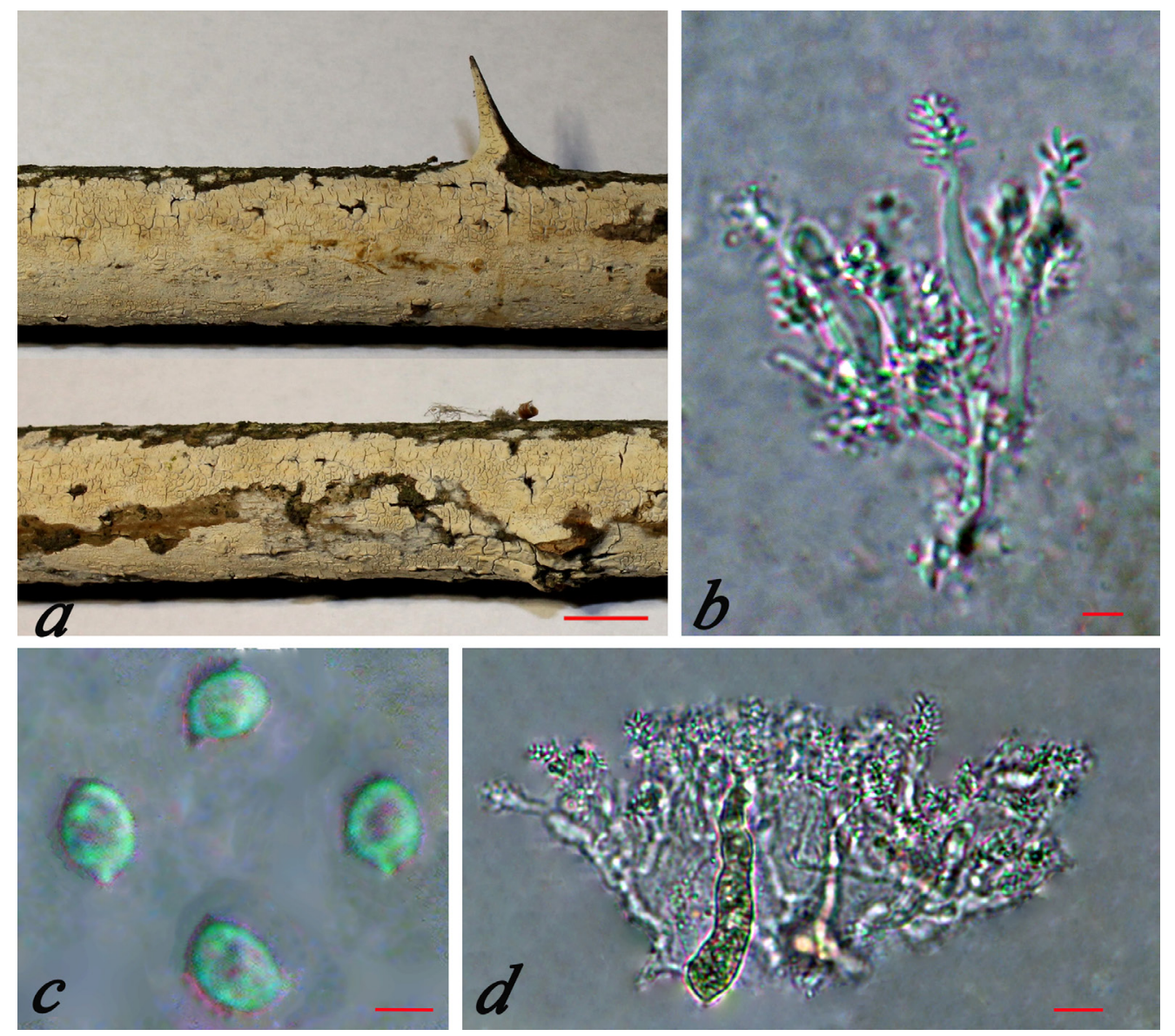

Fig. 2. Aleurodiscus dextrinoideocerussatus $K W$-M 70873: $a$ - general view of basidioma; $b, d-$ hymenium with acanthophyses and gloeocystidia; $c$ - basidiospores (bars: $a-1 \mathrm{~cm}, b, d-20 \mu \mathrm{m}, c-5 \mu \mathrm{m}$ )

Distribution. Europe: France, Italy, Montenegro, Russia, Switzerland (Bernicchia, Gorjón, 2010). Asia: Russia (Zhukov, 1995). North America: Canada and the USA (Eriksson, Weresub, 1974; Gilbertson, Lindsey, 1989).

The species is a new record for Ukraine.

\section{Hymenochaetales Oberw.}

Hymenochaetaceae Donk

Tubulicrinis glebulosus (Fr.) Donk [as 'glebulosa'], Fungus, Wageningen 26(1-4): 14. 1956 (Fig. 1; c-e)

Basidioma resupinate, effused, 90-100 $\mu \mathrm{m}$ thick, hymenophore smooth, floccose to pilose under the lens by projecting cystidia, membranaceous, closely adnate to the substratum, hymenial surface whitish to pale ochraceous, margin indeterminate. Hyphal system monomitic. Hyphae hyaline, compactly arranged in subhymenium and trama, branching from or near clamps, $2.0-3.5 \mu \mathrm{m}$ in diameter, thin-walled in subhymenium, thin- to slightly thick-walled in trama, smooth, clamps present at all septa. Cystidia (lyocystidia) cylindrical, 50-120 × 5.5-9.0 $\mu \mathrm{m}$, thickwalled, with lumen, that gradually expanding towards the apex, some of them being encrusted with crystals in the terminal part, apex of lyocystidia thin-walled, narrowly obtuse; some lyocystidia slightly amyloid, apical thin-walled part of lyocystidia rapidly dissolving in $5 \% \mathrm{KOH}$. Basidia hyaline, clavate with a median constriction, $14-22 \times 4.5-5.0 \mu \mathrm{m}$, thin-walled, with four subulate sterigmata $(4.0-5.5 \times 0.7-0.8 \mu \mathrm{m})$. Basidiospores hyaline, cylindrical to slightly allantoid, in side view slightly bent, $6-7 \times 1.8-2.2 \mu \mathrm{m}$, thinwalled, smooth, with homogeneous contents, neither amyloid, nor dextrinoid or cyanophilous.

Specimens examined: Ukraine, Chernihiv Region, Ichnia District, Ichnia National Nature Park, July 17, 2016, outskirts of Peliukhivka village; September 16, 2016, outskirts of Khaienky village, Tsybanovka Stow, square 18. On fallen branches of Pinus sylvestris, $K W-M$ 70874, 70875. 
Ecological peculiarities: on fallen branches of angiosperms (Hallenberg, 1984; Maekawa, 2002; Zmitrovich, 2003) and gymnosperms (Bernicchia, Gorjón, 2010).

Distribution. Europe: Austria, Albania, Belarus, Belgium, Bosnia and Herzegovina, Croatia, Estonia, Finland, France, Germany, Greece, Italy, Macedonia, Norway, Portugal, Russia, Slovenia, Spain, Sweden, Switzerland, United Kingdom (Bernicchia, Gorjón, 2010). Asia: Russia (Zmitrovich, 2003; Viner et al., 2016), China (Maekawa, 2002), Taiwan (Lin, Chen, 1990), Turkey (Ghobad-Nejhad et al., 2009). Africa: Morocco (Tellería et al., 2016). North America: Canada (Hallenberg, 1984), Mexico (Spirin, Ryvarden, 2016), the USA (Martin, Gilbertson, 1977). South America: Argentina (Rajchenberg, 2002).

The species is a new record for Ukraine.

Russulales Kreisel ex P.M. Kirk, P.F. Cannon \& J.C. David

\section{Stereaceae Pilát}

Aleurodiscus dextrinoideocerussatus Manjón,

M.N. Blanco \& G. Moreno, in Moreno, Blanco \& Manjón, Mycotaxon 39: 351. 1990 (Fig. 2)

Basidioma resupinate, effused, waxy when fresh, cracked after drying, hymenial surface is smooth to slightly tuberculate, whitish, cream to pale yellowish, margin clearly differentiated. Hyphal system monomitic, generative hyphae with clamps, hyaline, thin-walled, 2.0-3.5 $\mu \mathrm{m}$ wide. Acanthophyses abundant, basal smooth, about $30 \times 3.0-3.5 \mu \mathrm{m}$, apically with numerous protuberances and branches with indextrinoid reaction in the apical part. Gloeocystidia cylindrical, fusiform to moniliform with several constrictions, $70-100 \times$ 8-10 $\mu \mathrm{m}$, with yellowish granular content. Basidia subclavate $45-50 \times 6-7 \mu \mathrm{m}$, with four sterigmata and with a basal clamp. Basidiospores subglobose-subovoid with small apiculus, 7.0-7.5 × 4.2-5.0 $\mu \mathrm{m}$, smooth, thin-walled, slightly amyloid.

Remarks: both samples from Ukraine, the one found in Ichnia National Nature Park and another in Luhansk Nature Reserve (Ordynets et al., 2013), show no acanthophyses dextrinoid reaction. Moreover, they have subglobose-subovoid basidiospores, unlike the original description, where the spores thereof are of ellipsoid-subovoid shape.

Specimens examined: Ukraine, Chernihiv Region, Ichnia District, Ichnia National Nature Park, August 06, 2016, Kuty Stow, square 42. On attached branch of dead tree of Robinia pseudoacacia L., KW-M 70873.
Ecological peculiarities: on decayed wood, preferably of deciduous trees but may also occur on coniferous wood (Núñez, Ryvarden, 1997; Boidin, Gilles, 2001; Ghobad-Nejhad et al., 2008; Ordynets et al., 2013; Urbizu et al., 2014; Tellería et al., 2016).

Distribution. Europe: Croatia, France, Italy, Portugal, Spain, Ukraine (Bernicchia, Gorjón, 2010). Asia: Iran (Ghobad-Nejhad et al., 2008), Nepal (Núñez, Ryvarden, 1997). Africa: Morocco (Tellería et al., 2016). North America: Mexico (Urbizu et al., 2014).

According to A. Bernicchia and S. Gorjón (Bernicchia, Gorjón, 2010), it is a rare species all over Europe, recorded from some Mediterranean countries. In Ukraine, this species was reported only once from Luhansk Nature Reserve (Ordynets et al., 2013).

\section{REFERENCES}

Akulov A.Yu., Usichenko A.S., Leontyev D.V., Yurchenko E.O., Prydiuk M.P. Annotated checklist of aphyllophoroid fungi of Ukraine. Mycena, 2003, 2(2): $1-76$.

Bernicchia A., Gorjón, S.P. Corticiaceae s. 1. In: Fungi Europaei. Ed. S.V. Alassio. Italia: Candusso Edizioni, 2010, vol. 12, 1008 pp.

Boidin J., Gilles G. Corticiés s. 1. intéressants ou nouveaux pour la France (Basidiomycotina). Bull. Soc. Mycol. France, 1990, 106(4): 135-167.

Boidin J., Gilles G. Basidiomycètes Aphyllophorales de l'ile de Réunion XXIII - Aleurodiscoideae. Bull. Soc. Mycol. France, 2001, 117(3): 173-181.

Eriksson J., Ryvarden L. Aleurodiscus - Confertobasidium. In: The Corticiaceae of North Europe. Oslo: Fungiflora, 1973, vol. 2, pp. 60-286.

Eriksson J., Weresub L.K. Amylocorticium canadense (Burt) John Eriksson \& Weresub. Fungi Canadenses, 1974, 45: 2.

Ghobad-Nejhad M., Hallenberg N., Kotiranta H. Additions to the corticioids of the Caucasus from NW Iran. Mycotaxon, 2008, 105: 269-293.

Ghobad-Nejhad M., Hallenberg N., Parmasto E., Kotiranta H. A first annotated checklist of corticioid and polypore basidiomycetes of the Caucasus region. Mycol. Balcanica, 2009, 6: 123-168.

Gilbertson R.L., Lindsey J.P. North American species of Amylocorticium, a genus of brown rot fungi. Memoir. New York Bot. Gard., 1989, 49: 138-146.

Hallenberg N. Compatibility between species of Corticiaceae s. 1. from Europe and North America. Mycotaxon, 1984, 21: $335-388$.

Heluta V.P. Flora Gribov Ukrainy. Muchnistorosianyie griby. Kyiv: Naukova Dumka, 1989, 256 pp. [Гелюта В.П. Флора грибов Украины. Мучнисторосяные грибы. Киев: Наук. думка, 1989, 256 с.].

Jülich W., Stalpers J.A. The resupinate non-poroid Aphyllophorales of the Northern Hemisphere. New York: North-Holland Publ. Comp., 1980, 335 pp. 
Küffer N., Lovas P.S., Senn-Irlet B. Diversity of woodinhabiting fungi in natural beech forests in Transcarpathia (Ukraine): a preliminary survey. Mycol. Balcanica, 2004, 1: 129-134.

Lin S.H., Chen Z.C. The Corticiaceae and the resupinate Hydnaceae of Taiwan. Taiwania, 1990, 35(2): 69-111.

Maekawa N. Corticioid fungi (Basidiomycetes) collected in Sichuan province, China. Mycotaxon, 2002, 83: 81-95.

Martin K.J., Gilbertson R.L. Synopsis of wood-rotting fungi on spruce in North America. 1. Mycotaxon, 1977, 6(1): 43-77.

Mueller G.M., Schmit J.P., Leacock P.R., Buyck B., Cifuentes J., Desjardin D.E., Halling R.E., Hjortstam K., Iturriaga T., Larsson K.-H., Lodge D.J., May T.W., Minter D., Rajchenberg M., Redhead S.A., Ryvarden L., Trappe J.M., Watling R., Wu Q. Global diversity and distribution of macrofungi. Biodivers. Conserv., 2007, 16(1): 37-48.

Núñez M., Ryvarden L. The genus Aleurodiscus (Basidiomycotina). Synopsis Fungorum, 1997, 12: 1-164.

Ordynets O.V., Akulov O.Yu. Studia Biologica, 2011, 5(3): 109-124. [Ординець О.В., Акулов О.Ю. Афілофороїдні гриби відділення "Крейдова флора" Українського степового природного заповідника. Біол. сmydiï, 2011, 5(3): 109-124].

Ordynets O., Akulov O., Helleman S. Chornomors'k. bot. z., 2013, 9 (1): 57-83. [Ординець О., Акулов О., Хеллеман С. Перші відомості про різноманіття грибів відділення "Трьохізбенський степ" Луганського природного заповідника. Чорноморськ. бот. жж., 2013, 9(1): 57-83].

Ordynets O.V., Akulov O.Yu., Shyian-Hlotova H.V. Nature Reserves in Ukraine, 2011, 17(1-2): 28-33. [Ординець О.В., Акулов О.Ю., Шиян-Глотова Г.В. Афілофороїдні гриби Станично-Луганського відділення Луганського природного заповідника. Запов. справа в Україні, 2011, 17(1-2): 28-33].

Ordynets O.V., Yurchenko E.O. Ukr. Bot. J., 2010, 67(5): 725-735. [Ординець О.В., Юрченко Є.О. Нові та маловідомі для України види кортиціоїдних грибів. Укр. бот. жсурн., 2010, 67(5): 725-735].
Rajchenberg M. Corticioid and polyporoid fungi (Basidiomycotina) that decay Austrocedrus chilensis in Patagonia, Argentina. Mycotaxon, 2002, 81: 215-27.

Shevchenko M.V. Ukr. Bot. J., 2017, 74(3): 293-297. [Шевченко М.В. Нові та рідкісні для України види кортиціоїдних грибів. Укр. бот. жжурн., 2017, 74(3): 293-297].

Spirin V., Ryvarden L. Some basidiomycetes (Aphyllophorales) from Mexico. Synopsis Fungorum, 2016, 35: 34-42.

Tellería M.T., Dueñas M., Melo I., Salcedo I., Cardoso J., Fernández-López Javier, Martín M.P. Corticioid fungi (Basidiomycota) from the Biosphere Reserve of Arganeraie, Morocco: a preliminary survey. Nova Hedwigia, 2016, 103(1-2): 193-210.

Urbizu M., Siqueiros M.E., Abrego N., Salcedo I. New records of aphyllophoroid fungi from Aguascalientes, Mexico and an approach to their ecological preferences. Revista Mexicana de Biodiversidad, 2014, 85: 1007-1018.

Usichenko A.S. Chornomors'k. bot. z., 2009, 5(2): 276-289. [Усіченко А.С. Нові знахідки афілофороїдних грибів 3 Північного Сходу України. Чорноморськ. бот. же., 2009, 5(2): 276-289].

Viner I.A., Schigel D.S., Kotiranta H. New occurrences of aphyllophoroid fungi (Agaricomycetes, Basidiomycota) in the Central Forest State Biosphere Nature Reserve, Tver Region, Russia. Folia Cryptogam. Estonica, 2016, 53: 81-91.

Yurchenko E.O. Nature substrata for corticioid fungi. Acta Mycol., 2006, 42(1): 113-124.

Zhukov E.A. Aphyllophorales (Basidiomycetes) from Central Siberia. Mycotaxon, 1995, 53: 437-445.

Zhyhalenko O.A. Ukr. Bot. J., 2009, 66(6): 836-845. [Жигаленко О.А. Лісова рослинність Ічнянського національного природного парку. Укр. бот. жмурн., 2009, 66(6): 836-845].

Zmitrovich I.V. Tremelloid, aphyllophoroid and pleurotoid Basidiomycetes of Veps Plateau (Northwest Russia). Karstenia, 2003, 43(1): 13-36.

Zmitrovich I.V. Poriadok afilloforovyie. In: Opredelitel gribov Rossii. Moscow; St. Petersburg: KMK, 2008, issue 3, 278 рр. [Змитрович И.В. Порядок афиллофоровые. В кн.: Определитель грибов России. М.; СПб: КМК, 2008, вып. 3, 278 с.].

Recommended for publication by V.P. Hayova
Submitted 24.01.2018 
Шевченко М.В. Цікаві знахідки кортиціоїдних грибів в Ічнянському національному природному парку. Укр. бот. журн., 2018, 75(1): 77-83.

Інститут ботаніки ім. М.Г. Холодного НАН України вул. Терещенківська, 2, Київ 01004, Україна

Кортиціоїдні гриби належать до різних порядків класу Agaricomycetes (Basidiomycota) і складають гетерогенну за походженням групу грибів. В Україні, за сучасними даними, відомо близько 280 видів грибів цієї групи. У порівнянні з іншими країнами Європи, видове різноманіття кортиціоїдних грибів України досліджено значно гірше. Матеріалами для цієї статті були зразки, зібрані протягом червня-жовтня 2016 р. та березня-жовтня 2017 р. у лісових угрупованнях Ічнянського національного природного парку (Ічнянський р-н, Чернігівська обл., Україна). На основі аналізу власних гербарних матеріалів в районі дослідження нами було виявлено 90 видів кортиціоїдних грибів. У статті наведені дані про цікаві знахідки трьох видів грибів. Два із них (Amylocorticium canadense та Tubulicrinis glebulosus) - нові для України, один (Aleurodiscus dextrinoideocerussatus) є рідкісним як в Україні, так і у світі, вдруге виявлений на території країни і є новим для Лісостепової зони. Для дослідження мікроструктур виготовляли тимчасові мікропрепарати у 5\%-му водному розчині гідроксиду калію, реактиві Мельцера та бавовняному синьому у молочній кислоті. Для всіх видів подані описи досліджених зразків, що супроводжуються оригінальними фотографіями, інформацією про субстратну спеціалізацію та про поширення у світі. Зібрані зразки передано до Національного гербарію Інституту ботаніки ім. М.Г. Холодного НАН України $(K W-M)$.

Ключові слова: Україна, Лівобережний Лісостеп, Amylocorticiales, Hymenochaetales, Russulales, Aleurodiscus dextrinoideocerussatus, Amylocorticium canadense, Tubulicrinis glebulosus
Шевченко М.В. Интересные находки кортициоидных грибов в Ичнянском национальном природном парке. Укр. бот. журн., 2018, 75 (1): 77-83.

Институт ботаники им. Н.Г. Холодного НАН Украины ул. Терещенковская, 2, Киев 01004, Украина

Кортициоидные грибы принадлежат к разным порядкам класcа Agaricomycetes (Basidiomycota) и составляют гетерогенную по происхождению группу грибов. В Украине, по современным данным, известно около 280 видовгрибовэтойгруппы. Всравнениисдругимистранами Европы, видовое разнообразие кортициоидных грибов Украины исследовано значительно хуже. Материалами для настоящей статьи послужили образцы, собранные в июне-октябре 2016 г. и марте-октябре 2017 г. в лесных сообществах Ичнянского национального природного парка (Ичнянский р-н, Черниговская обл., Украина). На основе анализа собственных гербарных материалов в районе исследования нами было обнаружено 90 видов кортициоидных грибов. В статье приведены данные об интересных находках трех видов грибов. Два из них (Amylocorticium canadense и Tubulicrinis glebulosus) - новые для Украины, один (Aleurodiscus dextrinoideocerussatus) является редким как в Украине, так и в мире, второй раз обнаружен на территории страны и является новым для Лесостепной зоны. Для исследования микроструктур изготавливали временные микропрепараты в 5\%-ном водном растворе гидроксида калия, реактиве Мельцера и хлопчатобумажном синем в молочной кислоте. Для всех видов представлены описания исследованных образцов, сопровождающиеся оригинальными фотографиями, информацией о субстратной специализации и о распространении в мире. Собранные образцы переданы в Национальный гербарий Института ботаники им. Н.Г. Холодного НАН Украины $(K W-M)$.

Ключевые слова: Украина, Левобережная Лесостепь, Amylocorticiales, Hymenochaetales, Russulales, Aleurodiscus dextrinoideocerussatus, Amylocorticium canadense, Tubulicrinis glebulosus 